\title{
Attention Deficit Hyperactive Disorder (ADHD) Impact Upon Community Health from a Pediatric Endocrine Perspective George Moll*
}

Department of Pediatrics and Pediatric Endocrinology, University of Mississippi Medical Center, USA

\begin{abstract}
A primary goal of Pediatricians and especially Pediatric endocrinologists is to support healthy physical growth and mental development of children. The National Institute of Mental Health reports one in five (21\%) children have diagnosable mental, emotional or behavioral disorders with Attention Deficit/hyperactivity (ADHD) most prevalent. A 2010 survey finds five million US children 3-17 years of age diagnosed ADHD (8\%). With appropriate treatment, children with ADHD can improve short term learning that raises concerns for non-prescribed use such as during college finals and controversy regarding ADHD therapy addiction and substance abuse epidemic. In a University Mississippi Medical Centre, Pediatric endocrine practice I note 225 of $5-10$ years old patients $(\sim 8 \%)$ to be diagnosed and treated outside that practice for ADHD. The ADHD literature is reviewed for influence upon Pediatric endocrine therapy for autoimmune thyroiditis (56 of 225), hyperthyroidism (3), diabetes mellitus type 1 (9), congenital adrenal hyperplasia (2) and the majority for various physical growth disorders (180). Two case report summaries are presented where ADHD consideration delayed endocrine diagnoses. However, ADHD therapy is noted to minimally interfere with thyroid, diabetes mellitus or adrenal therapy though individual compliance with prescribed endocrine therapy can be adversely affected. Long term ADHD therapy is noted to delay physical growth consistent with literature reported 6-year persistent delayed "catch-up" growth. ADHD children are encouraged to attend to monitoring for appropriate ADHD therapy adjustments with their prescribing physicians. These observations encourage attention to 4-6 mon interval growth assessments for children, especially those entering puberty on ADHD therapy, to consider early growth therapy to optimize individual adult height attainment.
\end{abstract}

Keywords: Attention deficit hyperactive disorder; Thyroid; Diabetes mellitus; Adrenal; Physical growth; CNS stimulants

\section{Introduction}

Attention Deficit Hyperactive Disorders (ADHD) is a growing concern within our multi-media, multi-tasking contemporary society. Quality improvement is a major focus for both career development and healthcare. Healthy physical growth and development is central to Pediatric endocrinology and Pediatric practice. The rising occurrence of ADHD likely diagnosed and treated by behavioral specialists and Pediatricians should not direct attention from diagnosis and treatment of metabolic hormone imbalance that may also contribute to abnormal behavior and poor academic performance. Distribution of 2005-2006 single attending physician patient clinic visits and Pediatric endocrine diagnoses is shown in Table 1. Review of the impact of ADHD within this Pediatric endocrine practice is necessary to appreciate potential therapy interactions.

\begin{tabular}{|c|c|}
\hline \multicolumn{2}{|c|}{ A pediatric endocrinologist's one year patient activity census } \\
\hline $\begin{array}{c}\text { Diabetes } \\
\text { Mellitus }\end{array}$ & 760 Clinic patient visits and 110 in-patient events \\
\hline Thyroid & 36 Congenital Hypothroid \\
\hline & 10 Hyperthyroid \\
\hline $\begin{array}{c}\text { Physical } \\
\text { growth }\end{array}$ & 840 Goiter \& other thyroid \\
\hline & 300 Puberty (200 delayed and 100 precocious) \\
\hline & 30 835 short and 5 tall) \\
\hline & 30 Pituitary concerns (hypo-, adenoma, tumors) \\
\hline & 30 Calcium/Bone concerns \\
\hline $\begin{array}{c}\text { In-patient/ } \\
\text { Consult } \\
\text { concerns }\end{array}$ & $\begin{array}{c}80 \text { covering all aspects of physical growth and development, } \\
\text { often BG concerns and pituitary hormone/metabolic assessment }\end{array}$ \\
\hline
\end{tabular}

Table 1: A pediatric endocrinologist's one year patient activity census.

\section{ADHD}

National Institute of Mental Health review reports 13\%-20\% of children living in the United States experience a serious mental disorder in a given year among which diagnosable mental, emotional or behavioral disorders including ADHD are most prevalent $[1,2]$. Diagnostic and Statistical Manual of Mental Disorders (DSM-5, 2013) criteria for diagnosis of ADHD divide ADHD into primarily Inattention (9 receptive behavior directed questions), primarily Hyperactivity/Impulsivity (9 distracted behavior directed questions) or Mixed Inattention \& Hyperactivity/Impulsivity (Table 2). To be diagnosed in one of these categories a child or adolescent up to 16 years of age must fit at least 6 (adults 5) questions or more in Inattentive or at least 6 (adults 5) questions or more in Hyperactivity/Impulsivity or combined categories total at least 6 or more tested on 2 or more occasions in 2 or more settings. The ADHD behavior must have notable detracting effects upon an individual's social, academic or occupational performance not explainable by another mental disorder [3]. Applying DSM-5 criteria, children with ADHD generally divide into $10-15 \%$ Inattentive type, $3 \%$ hyperactive/impulsive type and $85 \%$ mixed or combined type [4].

DSM-5 criteria place ADHD disorders within the conceptual framework of autism spectrum disorders. The National Institute of Mental Health (NIMH) reports that in 2010 there were five million

*Corresponding author: George Moll, MD, Ph.D., Tenured Professor, Department of Pediatrics and Pediatric Endocrinology, University of Mississippi Medical Center 2500 North State Street, Jackson MS, 39216, USA, Tel: 601-672-2313; E-mail: gmoll1220@gmail.com

Received: June 27, 2018; Accepted: July 10, 2018; Published: July 17, 2018

Citation: Moll G (2018) Attention Deficit Hyperactive Disorder (ADHD) Impact Upon Community Health from a Pediatric Endocrine Perspective. Prim Health Care 8: 301. doi: 10.4172/2167-1079.1000301

Copyright: (c) 2018 Moll G. This is an open-access article distributed under the terms of the Creative Commons Attribution License, which permits unrestricted use, distribution, and reproduction in any medium, provided the original author and source are credited. 


\begin{tabular}{|c|c|}
\hline Inattention & Hyperactivity/lmpulsivity \\
\hline $\begin{array}{c}\text { 1) Lack of attention to details/careless } \\
\text { mistakes }\end{array}$ & 1) Fidgetiness/squirms in seat \\
\hline 2) Difficulty maintaining attention & 2) Leaves seat frequently \\
\hline 3) Does not appear to listen & 3) Running about/seems restless \\
\hline $\begin{array}{c}\text { 4) Doesn't complete instructions (easily side- } \\
\text { tracked) }\end{array}$ & 4) Excessively loud/noisy \\
\hline 5) Difficulty organizing activities/tasks & 5) Continuously "on the go" \\
\hline 6) Avoids sustained mental effort & 6) Talks excessively \\
\hline 7) Misplaces and loses objects & 7) Blurts out answers \\
\hline 8) Easily distracted & 8) Difficulty waiting turn \\
\hline 9) Forgetful in daily activities & 9) Tends to act without thinking \\
\hline
\end{tabular}

Table 2: DSM-5 criteria for ADHD diagnoses.

children or adolescents 3-17 years of age (about $8 \%$ of the general population) diagnosed with ADHD. National Survey of Children's Health (NSCH) data (Table 3 ) show a rising trend in prevalence of US children 4-17 years of age ever diagnosed with ADHD with about a 2 -fold higher prevalence for boys than for girls [5].

The NSCH data indicate a higher trend of children 5-17 years of age ever diagnosed with ADHD (Table 3) in the US South, US Midwest and US Northeast while US West remained relatively stable at about 5\% [6]. A report on ADHD occurrence at 7 years of age noted $10 \%$ occurrence in children with history of low birthweight $v s .4 \%$ occurrence without low birthweight [7]. An inheritance pattern for ADHD is also noted with $70 \%-80 \%$ monozygotic twins both having ADHD if one is diagnosed with ADHD, 30-40\% fraternal twins both having ADHD if one is diagnosed with ADHD, $25 \%$ of children diagnosed ADHD have a sibling with ADHD and 15\%-40\% chance of ADHD child having a parent with ADHD [8-11]. This may explain some of the higher trend in US South with Mississippi ranking high among US States reporting low birthweights (11.4\% with $13.6 \%$ premature deliveries in 2017 population survey) and also challenged with need to improve Public School test results raising awareness for ADHD diagnosis [12,13].

ADHD medications are generally CNS catecholamine neurotransmitter pathway modifiers with CNS regional differential effects. A 2018 updated systematic review of the literature entitled Attention Deficit Hyperactivity Disorder: Diagnosis and Treatment in Children and Adolescents is available as an internet downloadable book through PubMed Health [14].

Worldwide the consumption of ADHD medications is rising with 2-fold methylphenidate US consumption from about 3.5\% 1996-1998 to about 7\% 2006-2008 [15]. This trend raises concern for guidelines to assist appropriate application of ADHD diagnosis and treatment and reduce addiction potential. The American Academy of Pediatrics (AAP) published clinical practice guidelines in 2011 to encourage incorporation of behavioral therapy as well as consideration of medical therapy: 4-5 year-old emphasize behavioral therapy and then consider methylphenidate as essentially only age group FDA approved ADHD therapy, 6-11 year-old FDA approved therapy starting with methylphenidate-like stimulant agents but also incorporate behavioral therapy and 12-18 year old FDA approved therapy with Assent and again incorporate behavioral therapy [4]. Tables of FDA approved ADHD medications can be found on internet (most approved for age and weight adjusted dose in 6 year-old or older and none approved for younger than 6 years of age) which are primarily stimulants as DEA Schedule II agents [16].

The NIH reports drug addiction/deaths to have risen about 2-fold for both men and women in the US from 2002 to 2015 when total

\begin{tabular}{|c|c|c|c|}
\hline \multicolumn{4}{|c|}{ NSCH Survey Prevalence ADHD Trends in US Children } \\
\hline NSCH Survey Year & 2003 & 2007 & 2011 \\
\hline ADHD in Survey Population & $7.80 \%$ & $9.50 \%$ & $11.00 \%$ \\
\hline ADHD in Survey Boys & $11.00 \%$ & $13.20 \%$ & $15.10 \%$ \\
\hline ADHD in Survey Girls & $4.40 \%$ & $5.60 \%$ & $6.70 \%$ \\
\hline \multicolumn{4}{|c|}{ Regional Prevalence ADHD Trends in US Children } \\
\hline NSCH Surveyed Years & $1998-2000$ & $2007-2009$ & \\
\hline US South & $8.10 \%$ & $10.30 \%$ & \\
\hline US Midwest & $7.10 \%$ & $10.20 \%$ & \\
\hline US Northeast & $5.70 \%$ & $8.60 \%$ & \\
\hline US West & $5.40 \%$ & $5.80 \%$ & \\
\hline
\end{tabular}

Table 3: NSCH survey prevalence ADHD trends in US children.

\begin{tabular}{|c|c|}
\hline Hyperthyroid Pertinent Symptoms & Hypothyroid Pertinent Symptoms \\
\hline Goiter & Goiter \\
\hline Eye prominence & Easy Fatigue \\
\hline Nervousness & Muscle hypertrophy but weak \\
\hline Increased appetite & Variable appetite \\
\hline Weight loss & Variable increase/decrease weight \\
\hline Emotional lability & Emotional instability \\
\hline
\end{tabular}

Table 4: Thyroid Pertinent Clinical Symptoms.

50,000 deaths involving overdose of all drugs [17]. Mississippi 2017 statistics with estimated population 2,984,100 dispersed over 48,430 square miles indicate overall total overdose opioid-like deaths for the year at 256 [18]. A 2014 Swedish Registry Study of those born 19601998 reports no indication of increased risk of substance abuse among ADHD stimulant treated patients [19]. However, a 2004 Canadian British Columbia study pointed out need for consideration of economic and child health-related quality of life impact upon a family with an ADHD treated child [20]. A 2014 Massachusetts General Hospital literature review concluded the data remain unsettled but ADHD pharmacotherapy by itself does not increase risk for substance use disorders (SUD) [21].

A 5 year review of a single attending Pediatric Endocrine University of Mississippi Medical Center (UMMC) physician's practice identified 225 patients diagnosed and treated for ADHD by healthcare specialists outside Pediatric endocrinology prior to or after referral to UMMC. The Pediatric Endocrine referrals were made for thyroid disorders, diabetes mellitus, adrenal disorders and calcium disorders.

\section{ADHD and thyroid disorders}

Among the 225 patients with ADHD 56 (25\%) were being treated for autoimmune thyroiditis. Among 100 patients being treated for hyperthyroidism 3 (3\%) presented with ADHD. Symptoms of thyroid dysfunction overlap with ADHD mental alertness and behavioral concerns as listed in Table 4.

A study of 227 children diagnosed with ADHD found 14 (5.4\%) had thyroid abnormalities to a greater extent than estimated occurrence of thyroid test abnormalities in a comparable general population of children $(\leq 1 \%)$. None of the 227 children were found to have Global Thyroid Resistance (GTR) but a literature review found an estimated $46 \%$ of GTR patients to also have the diagnosis of ADHD [22]. A study of 43 children with ADHD and 28 age and gender matched controls found no significant differences for thyroid test results with or without ADHD [23].

Psychiatric unit patient admissions tend to have thyroid tests. A published chart review of 150 adolescent first-time admissions to a 
psychiatric unit who had thyroid tests found only mild thyroid test abnormalities that returned normal upon repeat testing. The authors concluded routine psychiatric unit admission thyroid testing was unnecessary. However, the authors agreed thyroid testing should be done if admission history and physical noted goiter or clinical symptoms of thyroid dysfunction [24]. Thyroid test series should include thyroid stimulating hormone (TSH) and Free Thyroxine levels as there may be serious CNS abnormality or tumor interference with hypothalamic/pituitary signaling.

\section{ADHD and diabetes mellitus}

Among 29 boys and 31 girls of 5-10 years of age with UMMC diagnosed diabetes mellitus type 1 (T1DM) five boys (17\%) and four girls (13\%) were diagnosed with ADHD after diagnosis with T1DM. These ADHD patients with T1DM were noted to require more attention to T1DM control with upward trend in their average blood sugar (BG) related hemoglobin Alc (HA1c) after start of their ADHD medical therapy.

Psychometric medications can interfere with blood sugar control. ADHD directed methylphenidate therapy can create susceptibility to develop acute pancreatitis as a cause of hyperglycemia. A 1999 Harvard review of this association with hyperglycemia noted one occurrence with methylphenidate (Ritalin) [25]. Anti-psychotic therapy can associate with hyperglycemia and rarely diabetic ketoacidosis (DKA) or hyperosmolar hyperglycemic syndrome (HHS) possibly through a postulated impaired insulin gene expression similar to that seen in Maturity Onset Diabetes Mellitus of Youth type 3 [26]. A 13 year-old male with T1DM and ADHD required admission for anti-psychotic behavior therapy and was noted to have BG over $200 \mathrm{mg} / \mathrm{dL}$ during 6 days of risperidone therapy that returned to BG less than $200 \mathrm{mg} /$ $\mathrm{dL}$ within 36 hours after stopping risperidone [27]. However, appetite suppressive and metabolic effects of ADHD medical therapy may more often associate with occurrence of hypoglycemia. Methylphenidate is a potent inhibitor of oral hypoglycemic agent liver metabolizing cytochrome P2C9 that one report associated with hypoglycemia events in a diabetes mellitus type 2 patient taking oral glipizide [28].

\section{ADHD and adrenal}

Adrenal function is responsive to emotional and physical stress upon which ADHD should have a strong influence. Among UMMC congenital adrenal hyperplasia patients only 2 (about 5\%) were also diagnosed to have ADHD. A 2011 published report studied hypothalamic-pituitary-adrenal (HPA) axis in 128 males 6-14 years of age compared with 30 healthy male controls under overnight rest conditions. The ADHD patients were divided among Inattention type (ADHD-I, $\mathrm{n}=44$ ), Hyperactive/Impulsive type (ADHD-HI, $\mathrm{n}=32$ ) and Combined type (ADHD-C, $n=52$ ). All groups and controls had comparable ACTH levels. The plasma cortisol levels were significantly lower $(p<0.001)$ for the total ADHD and all subgroups of ADHD relative to the controls: $154.36+/-71.62$ (SD) to $258.30+/-136.39$ $\mathrm{nmol} / \mathrm{L}$ vs. $384.53+/-141.43 \mathrm{nmol} / \mathrm{L}$. The impression was that of ADHD creating an under-reactive HPA in the absence of stress [29].

Another test of the HPA axis was performed with 68 ADHD (54 boys and 14 girls, mean age 8.8 years) by measuring saliva cortisol levels pre- and again post- psychological testing. The majority of tested children, 51 of the 68 , noted no cortisol rise after testing. The authors concluded there was significant blunting of the cortisol response to psychological stress with $7.4 \%$ and $9.5 \%$, respectively, decreased verbal and total IQ [30].

\section{ADHD and physical growth}

The majority of UMMC Pediatric endocrine clinic patients have physical growth concerns. Among these patients 180 (about 10\%) were noted to also have a diagnosis of ADHD. Long-term ADHD therapy is known to be suppressive upon physical growth.

The first 6 years of physical growth of 410 children treated with central stimulants for ADHD or ADD in an observational study at Centre for Child and Adolescent Psychiatry, Aarhus University Hospital, Denmark was published in 2015 [31]. The observed cohort consisted of 368 male and 42 female children who entered the study at $9.2+/-2.4(\mathrm{SD})$ years of age with weight significantly $(p<0.0001)$ above the mean at $\mathrm{z}$-score 0.59 and height significantly $(p<0.001)$ above the mean at $\mathrm{z}$-score 0.21 . Weight and height $\mathrm{z}$-scores significantly $(p<0.01)$ declined at any time interval out to 6 years. All participants experienced relative reduction in body weight and temporary halt in growth to near average for age at 1 to 4 years into the study. Thereafter, participants noted some regain in weight to mean $\mathrm{z}$-score about 0.12 at 6 years but continued to note a lack of regain in height with mean $\mathrm{z}$-score about -0.1 and no apparent rebound in height attainment out to 6 years. These results support the need for reasonable 4-6 month interval growth monitoring of patients on chronic ADHD medical therapy.

\section{ADHD and calcium imbalance as two case summaries}

Case 1 was a $65 / 6$ year-old male referred to his Primary Care Provider (PCP) for ADHD diagnosis and medical therapy when he was failing $1^{\text {st }}$ grade. After 6 mon of failed response to medical therapy blood tests were sent that returned with incidental hypercalcemia 13.5 $\mathrm{mg} / \mathrm{dL}$ for which he was referred to UMMC Pediatric Endocrine clinic.

His medical history indicated he had been seen for possible Kawasaki disease with fever at 2 years of age when his serum calcium was $12.5 \mathrm{mg} / \mathrm{dL}$ but not followed-up at that time. His father was outside the family at 48 years of age with question of calcium imbalance but non-compliant with prescribed T2DM care. On physical exam his height and weight were $25^{\text {th }}$ percentiles, Temperature $97.8^{\circ} \mathrm{F}$, pulse 70 , respirations 22 and BP 109/72. He appeared appropriate for age with grossly intact cranial nerves, symmetric average good strength and normal gait. His work-up centered about relative hyperparathyroidism $62.1 \mathrm{pg} / \mathrm{ml}$ with hypercalcemia $13.3-13.8 \mathrm{mg} / \mathrm{dL}$ and ionized calcium $1.65 \mathrm{mmol} / \mathrm{L}$. His extensive work-up was otherwise normal except for low urine fractional calcium excretion ratio 0.006 (normal $>0.01$ ) consistent with the rare diagnosis of familial hypo-calcuric hypercalcemia. His genetic assessment returned known inactivating calcium sensing receptor (CASR) mutation which responded to a trial of Cinacalcet therapy awaiting long-term coverage for expense of Cinacalcet as his serum calcium levels are borderline $13-14 \mathrm{mg} / \mathrm{dL}$ and not so benign long-term [32].

Case 2 was a 13 year-old female with panic attacks for about 8 weeks, lasting a few minutes and particularly occurring in school that resulted in ADHD consideration and attempted behavioral therapy with Paxil failed after 3-4 mon. At that time her PCP obtained blood tests that returned serum calcium $5 \mathrm{mg} / \mathrm{dL}$ and phosphorous $11 \mathrm{mg} / \mathrm{dL}$ for which she was referred emergently for Pediatric Endocrine care of hypocalcemia.

Her medical history revealed some bad headaches and nonspecific somatic pain, poor primary teeth mineralization, recurrent/ persistent candidiasis and irregular sparse cycles following menarche 12 years of age. On physical exam, her height and weight were $20^{\text {th }}$ percentiles, temperature $98.3^{\circ} \mathrm{F}$, pulse 100 , respirations 28 and BP 
109/52. Slight positive Chvostek sign but otherwise normal neurologic exam and symmetric muscle strength. Her work-up centered upon hypoparathyroidism initial $<8 \mathrm{pg} / \mathrm{mL}$ and likely autoimmune. She responded well to IV calcium therapy and transferred to oral well. At 3 mon from hypoparathyroidism diagnosis she noted recurrent episodes of nausea and vomiting initially considered viral with afternoon cortisol $2.8 \mathrm{mcg} / \mathrm{dL}$, calcium $10.2 \mathrm{mg} / \mathrm{dL}$ and phosphorous $5.8 \mathrm{mg} / \mathrm{dL}$ with magnesium $1.0 \mathrm{mEq} / \mathrm{L}$. However, by 4 mon from diagnosis her calcium was unusually elevated $13.1 \mathrm{mg} / \mathrm{dL}$ with phosphorous $4.6 \mathrm{mg} / \mathrm{dL}$. Adrenal assessment then indicated primary adrenal insufficiency with $8 \mathrm{am}$ and $7 \mathrm{pm}$ cortisol $<1.0 \mathrm{mcg} / \mathrm{dL}$ but elevated Adrenocorticotropic hormone (ACTH) $8 \mathrm{am} 4180 \mathrm{pg} / \mathrm{mL}$ and $7 \mathrm{pm} 2920 \mathrm{pg} / \mathrm{mL}$. She also had premature ovarian failure. A genetic assessment for Autoimmune Polyglandular Syndrome type 1 was sent and confirmed Autoimmune Poly-endocrinopathy-candidiasis-ectodermal dystrophy (APECED) associated known mutation in Autoimmune Regulator (AIRE) gene [33].

\section{ADHD Summary and Conclusion}

ADHD and its treatment, often with CNS stimulants, by a behavioral specialist or PCP may appear with little advanced notice in about $3 \%$ to $16 \%$ of Pediatric Endocrine clinic patients. With rising concern for drug addiction primarily surrounding opioid addiction, physicians must be aware of refinements in diagnosis of ADHD and guidelines to always include behavioral management as well as medication consideration. Any consideration of ADHD diagnosis must have a preceding general health history and physical with appropriate baseline lab tests. The rapid evolution of electronic medical records should make it easier for all of an individual patient's physicians to become aware of a diagnosis of ADHD and medical therapy. Suppression of Physical growth and development appear to be major side effects of ADHD therapy to impact Pediatric endocrine patient care requiring attention to 3 to 6 mon interval growth assessments for early consideration of optimal growth therapy. However, other endocrine disorders can influence and be influenced by ADHD and its prescribed medical therapy.

Thyroid dysfunction symptoms overlap those of ADHD (Tables 2 and 4). Thyroid dysfunction occurs in about 5.4\% of ADHD patients and thyroid function tests should be part of any initial ADHD assessment along with adequate physical exam to detect goiters for early Pediatric Endocrine referral. Diagnosis of rare thyroid disorders such as Global Thyroid Resistance (GTR) in which as many as $46 \%$ of GTR patients can be diagnosed with ADHD that may delay their GTR diagnosis.

Diabetes mellitus type 1 and type 2 controls requires attention to daily prescribed and common sense routines that can be adversely affected by diagnosis and treatment of ADHD. ADHD therapy may lend itself to causing more insulin resistance with associated hyperglycemia but ADHD therapy associated appetite suppression and oral hypoglycemic agent reduced turnover may trend toward hypoglycemia. Patients with both ADHD and diabetes mellitus must be encouraged to follow medically necessary lifelong daily diabetes mellitus multiple BG daily monitoring and at least 3 to 4 mon interval diabetes mellitus clinical progress review.

Adrenal response to emotional and physical stress may be sluggish. Attention should be payed to unusually recurrent or persistent nausea and vomiting episodes as major symptoms for developing adrenal insufficiency particularly with difficult to explain hypercalcemia.

Rising prevalence of ADHD diagnosis and therapy, often performed by behavioral specialists and physicians outside a Pediatric Endocrine or other medical specialty practice, must be kept in mind and be part of an interval clinic medication update assessment. Patients may not consider ADHD diagnosis and therapy as potentially interacting with more physically directed medical concerns and patients must be directly queried as to ADHD diagnosis and therapy. A patient's ADHD diagnosis and therapy may significantly influence that patient's treatment program or that patient's enrolment in research protocols.

Funding Source: No funding was secured for this Health Surveillance review.

Conflict of Interest: The author has no conflicts of interest relevant to report to disclose.

\section{References}

1. Centers for Disease Control and Prevention (2016) Mental health surveillance among chjildren-united.

2. Merikangas KR, He JP, Brody D, Fisher PW, Bourdon K, et al. (2010) Prevalence and treatment of mental disorders among US children in the 2001-2004 NHANES. Pediatrics 125: 75-81.

3. Diagnostic and Statistical Manual of Mental Disorders, $5^{\text {th }}$ Edition (2013) DSM-5, American Psychiatric Association.

4. AAP Clinical Practice Guideline-ADHD (2011) Pediatrics 128:1-16.

5. The National Institute of Mental Health (2018) Attention-deficit/hyperactivity disorder (ADHD).

6. $\mathrm{CDC} / \mathrm{NCHS}$ (2018) Health data interactive and national health interview survey.

7. Treyvaud K, Ure A, Doyle LW, Lee KJ, Rogers CE, et al. (2013) Psychiatric outcomes at age seven for very preterm children: Rates and predictors. $J$ Child Psychol Psychiatry 54: 772-779.

8. Thapar A, Stergiakouli E (2008) An overview on the genetics of ADHD. Xin Li Xue Bao 40: 1088-1098.

9. Levy F, Hay DA, McStephen M, Wood C, Waldman I (1997) Attention-deficit hyperactivity disorder: A category or a continuum? Genetic analysis of a large-scale twin study. J Am Acad Child Adolesc Psychiatry 36: 737-744.

10. Faraone SV, Perlis RH, Doyle AE, Smoller JW, Goralnick JJ, et al. (2005) Molecular genetics of attention-deficit/hyperactivity disorder. Biol Psychiatry 57: 1313-1323.

11. Biederman J, Faraone SV, Mick E, Spencer T, Wilens T, et al. (1995) High risk for attention deficit hyperactivity disorder among children of parents with childhood onset of the disorder: a pilot study. Am J Psychiatry 152: 431-435.

12. Wolfe A (2017) Mississippi again unhealthiest state in the country. Clarion Ledger. Mississippi State Department of Health.

13. Mississippi Department of Education (2012) Mississippi testing task force.

14. Kemper AR, Maslow GR, Hill S, Namdari B, Allen LaPointe NM, et al. (2018) Attention Deficit Hyperactivity Disorder: Diagnosis and Treatment in Children and Adolescents. Rockville (MD): Agency for Healthcare Research and Quality (US). AHRQ Comparative Effectiveness Reviews.

15. International Narcotics Control Board (2009) Psychotropic substances.

16. McDonagh MS, Peterson K, Dana T, Thakurta S (2007) Drug class review on pharmacologic treatments for ADHD. Oregon Health and Science University.

17. Overdose Death Rates (2017) National institute on drug abuse.

18. The mississippi opioid and heroin data collaborative (2018) 2017 Provisional Data Report.

19. Chang Z, Lichtenstein P, Halldner L, D'Onofrio B, Serlachius E, et al. (2014) Stimulant ADHD medication and risk for substance abuse. J Child Psychol Psychiatry 55: 878-885.

20. Klassen AF, Miller A, Fine S (2004) Health-related quality of life in children and adolescents who have a diagnosis of attention-deficit/hyperactivity disorder. Pediatrics 114: e541-e547.

21. Zulauf CA, Sprich SE, Safren SA, Wilens TE (2014) The complicated relationship between attention deficit/hyperactivity disorder and substance use disorders. Curr Psychiatry Rep 16: 436-447. 
Citation: Moll G (2018) Attention Deficit Hyperactive Disorder (ADHD) Impact Upon Community Health from a Pediatric Endocrine Perspective. Prim Health Care 8: 301. doi: 10.4172/2167-1079.1000301

22. Weiss RE, Stein MA, Trommer B, Refetoff S (1993) Attention-deficit hyperactivity disorder and thyroid function. J Pediatr 123: 539-545.

23. Toren P, Karasik A, Eldar S, Wolmer L, Shimon I, et al. (1997) Thyroid function in attention deficit and hyperactivity disorder. J Psychiatr Res 31: 359-363.

24. Leo RJ, Batterman-Faunce JM, Pickhardt D, Cartagena M, Cohen G (1997) Utility of thyroid function screening in adolescent psychiatric inpatients. J Am Acad Child Adolesc Psychiatry 36: 103-111.

25. Cerulli TR, Alkoc SC, Salzman C (1999) Effects of psychotropic medications on pancreatic function: A review. Harv Rev Psychiatry 7: 54-60.

26. Bucholz S, Morrow AF, Coleman PL (2008) A typical antipsycholtic-induced diabetes mellitus: An update on epidemiology and postulated mechanism. Intern Med J 38: 603-606.

27. Stern AP, Trieu ML (2012) A case of antipsychotic-induced hyperglycemia in a child with insulin dependent diabetes mellitus. J Child Adolesc Psychopharmacology 22: 403-404.

28. Gontkovsky ST, Nevels R, McDonald NB, Winkelmann MH (2007) Decreased serum glucose levels after initiation of methylphenidate in a patient status post-cerebellar tumour resection a potential interaction with glipizide. Clin Drug Invest 27: 719-725.

29. Ma L, Chen YH, Chen H, Liu YY, Wang YX (2011) The function of hypothalamus-pituitary-adrenal axis in children with ADHD. Brain Res 1368: 159-162.

30. Shin DW, Lee SH (2007) Blunted hypothalamo-pituitary-adrenal axis reactivity is associated with the poor intelligence performance in children with attention-deficit/ hyperactivity disorder. Neuropediatrics 38: 298-303.

31. Powell SG, Frydenberg M, Thomsen PH (2015) The effects of longterm medication on growth in children and adolescents with ADHD: An observational study of a large cohort of real-life patients. Child Adolesc Psychiatry Ment Health 9: 50-62.

32. Lietman SA, Germain-Lee EL, Levine MA (2010) Hypercalcemia in children and adolescents. Curr Opin Pediatr 22: 508-515.

33. Guo Can-Jie, Leung PSC, Zhang Weici, Ma Xiong, Gershwin ME (2018) The immunology and clinical features of type 1 autoimmune Polyglandular syndrome (APS-1). Autoimmun Rev 17: 78-85. 